\title{
Mapeamento sistemático sobre conflitos, negociação e decisão em relacionamentos interorganizacionais
}

\author{
Barbara Beato Ribeiro ${ }^{1}$, Bruna Diirr ${ }^{1}$ \\ ${ }^{1}$ Universidade Federal do Estado do Rio de Janeiro (UNIRIO) - Rio de Janeiro - Brasil \\ \{barbara.ribeiro, bruna.diirr\}@uniriotec.br
}

\begin{abstract}
Organizations increasingly engage in interorganizational relationships to maximize results and reduce adversities. However, it is common to face conflicts, demands for negotiations, and subsequent decisionmaking when dealing with such social contracts. The purpose of this research is through a systematic literature mapping, identify conflicts, negotiations and decision making as well as improvements in the ongoing processes. The SLM results highlights the lack of unification in terms of the adopted processes and computational tools, thus enabling new research opportunities in this context.
\end{abstract}

Resumo. As organizações cada vez mais participam de relacionamentos interorganizacionais para maximizar seus resultados e reduzir suas adversidades. Contudo, é comum enfrentar conflitos, demandas por negociações e consequentes tomadas de decisão ao lidar com tais contratos sociais. A proposta desta pesquisa é, através de um mapeamento sistemático da literatura, identificar conflitos, negociações e tomadas de decisões nesses relacionamentos, bem como, melhorias nos processos em andamento. Os resultados do MSL sugerem a falta de uma unificação em termos de processos $e$ ferramentas computacionais adotadas, possibilitando assim novas oportunidades de pesquisa neste contexto.

\section{Introdução}

Atualmente, as organizações devem estar preparadas para trabalharem em conjunto, compartilhando resultados/dinâmicas, integrando processos/ferramentas, concordando em seus tópicos e com uma cultura que leve ao entendimento mútuo [Bocanegra et al. 2011, Legner e Wende 2007, Van Fenema et al. 2014, Zhu e Huang 2007]. Manter competitividade, atrair mais investidores, melhorar recursos, maximizar resultados e reduzir adversidades são alguns fatores que impulsionam os chamados relacionamentos interorganizacionais [de Castro et al. 2011, Diirr e Cappelli, 2018].

Relacionamentos interorganizacionais são estabelecidos quando organizações identificam problemas comuns e firmam um acordo específico para atingir um objetivo mútuo, minimizando seus problemas e maximizando seus resultados através do compartilhamento de habilidades, experiências, recursos humanos, financeiros, equipamentos, processos e informações [Samdantsoodol et al. 2012, Van Fenema et al. 2014]. Porém, apesar dos benefícios, ocorre a necessidade e dificuldade de trabalhar em conjunto, compartilhar informações e gerir compromissos entre as organizações envolvidas, o que pode levar a falhas no relacionamento interorganizacional [Bocanegra et al. 2011, Legner e Wende 2007, Van Fenema et al. 2014, Zhu e Huang 2007]. Os resultados de uma RSL sobre relacionamentos interorganizacionais [Diirr e Cappelli, 2018], apontam que existem vários desafios a serem superados nesse contexto, dentre os 
quais desafios relacionados à conflitos, tomada de decisão e negociações tanto entre as organizações quanto entre estas e seus clientes [Malakowsky e Kassick, 2014].

Este trabalho apresenta um Mapeamento Sistemático da Literatura (MSL) para melhor compreender onde essa relação pode falhar, o porquê e se isso é relacionado aos principais pontos que levam a uma tomada de decisão, as etapas e acontecimentos em uma negociação, bem como se estão relacionados a gerência dos conflitos no contexto da gestão de relacionamentos interorganizacionais. Identificam-se soluções, ferramentas computacionais e limitações que essas questões retornam às empresas de diferentes segmentos e o que está sendo feito para que esses processos, essenciais e diários, sejam menos burocráticos, mais benéficos e transparentes às organizações e seus funcionários. Além disso, apontam-se brechas na literatura, possibilitando futuras pesquisas na área.

Em busca na literatura, foram identificados trabalhos cujo enfoque está relacionado a esta pesquisa. Lopez et al. (2019) conduziram uma RSL para desenvolverem um framework que visa apoiar VEs (Virtual Enterprise) em termos de data information/business, além de melhorar a sinergia das empresas. Já Samdantsoodol et al. (2012) se pautaram em VEs dentro do chamado SCM (Supply Chain Management). Por fim, Troussier et al. (2010) realizou uma RSL para implementar "conceitos colaborativos emergentes" em organizações virtuais, que englobam, por exemplo, o apoio à "colaboração entre fornecedores para o desenvolvimento e fabricação de equipamentos de montagem". Apesar desses trabalhos detalharem RSL, eles apresentam um foco distinto da presente pesquisa, seja no contexto (por exemplo, estar focada em supply chain), seja em seu objeto de estudo (por exemplo, colaboração).

O artigo está estruturado da seguinte forma: A Seção 2 detalha o mapeamento sistemático realizado. A Seção 3 apresenta as questões de pesquisa, respondendo-as. Por fim, a Seção 4 reúne as considerações finais, benefícios obtidos, limitações identificadas e sugere possibilidades de aprofundamento do assunto.

\section{Mapeamento Sistemático}

O mapeamento sistemático da literatura (MSL) é uma metodologia que permite selecionar, interpretar e sumarizar o que a literatura dispõe para dado tópico [Kitchenham 2004; Biolchini et al. 2005]. O presente MSL, realizado em mar-jul/2020, seguiu o processo definido por Kitchenham (2004), como detalhado na Figura 1.

Primeiramente, o protocolo de mapeamento foi definido, garantindo que a análise seja abrangente, imparcial e repetível. Assim, foi identificada a questão de pesquisa ("O que foi discutido até então sobre conflito, tomada de decisão e negociação em relacionamentos interorganizacionais?"), as bases utilizadas (Web of Science, Scopus, IEEEXplore e ACM Digital Library) e os critérios de inclusão e exclusão. A abordagem PICO (Population, Intervention, Comparison, Outcome) ajudou na definição da string de busca aplicada nas bibliotecas digitais. Obteve-se um total de 416 artigos (publicados entre 1997 e 2020), que foram importados no Parsif.al, uma ferramenta de auxílio à condução de revisão sistemática. Retirou-se as duplicatas (28 artigos) e separou-se os artigos pelo critério de inclusão/exclusão através da leitura do abstract (47 artigos aceitos). Após isso, os artigos aceitos foram lidos integralmente, restando 33 artigos no MSL. Por fim, realizou-se o Backward Snowballing (Wholin, 2014), que analisa as referências de cada estudo pré-selecionado para obter mais estudos relevantes à pesquisa. Porém, não foram identificadas novas referências relevantes ao MSL. 


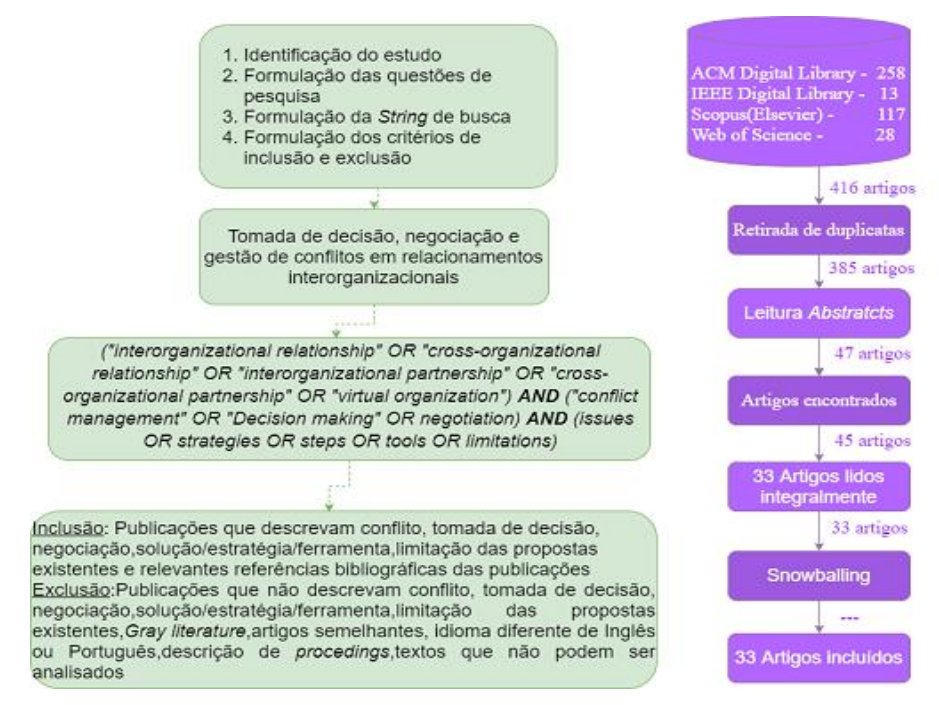

Figura 1. Planejamento, Execução e Resultado do MSL realizado

\section{Resultados Encontrados}

Os 33 artigos foram analisados, e as informações obtidas foram organizadas segundo 5 subquestões de pesquisa: Em um relacionamento interorganizacional: (QP1) Quais são os principais conflitos? (QP2) Como as tomadas de decisão são realizadas? (QP3) Como as negociações são realizadas? (QP4) Quais soluções/estratégias/ferramentas computacionais geralmente são adotadas? (QP5) Quais limitações existem?

Em relação aos conflitos, a literatura os define como "o desentendimento entre dois ou mais indivíduos, grupos ou empresas, que, de forma negativa causa discórdia e antipatia e, de forma positiva, motiva o aprendizado e a busca por novos desafios" [Griffin 2007 apud: Malakowsky e Kassick 2014] e como pontos de percepção de um assunto diferente (Robbins et al., 2010). Elliott e Scacchi (2003) discutem a utilização ou não de software não livre no apoio a ferramentas computacionais em uma organização virtual de desenvolvimento de software livre. Zhao et al. (2005) tratam da seleção de parceiros, um problema comum em organizações virtuais e de gestão de supply chain. Ollus et al. (2007) levantam que falta uma base sólida para a gestão e controle de organizações virtuais, citando o framework ECOLEAD (European Collaborative Networked Organisations Leadership Initiative) que apoia negociações, formação de contratos, seleção de novos parceiros e tomada de decisão em organizações virtuais. Carneiro et al. (2011) pautam que conflitos são comuns nas organizações virtuais (VO - Virtual Organization), o que atrasa os processos e desperdiça vantagens em participar de uma VO. No escopo de aprendizagem interorganizacional, Ganz (2018) tenta compreender, através de modelos, como as organizações utilizam e difundem o que aprendem. Tayles et al. (2005) examinaram 3 empresas de manufatura e como suas posições mudaram de "manufatura tradicional à virtual", estudando implicações e decisões estratégicas com base em informações financeiras.

Sobre as tomadas de decisão, entendidas na literatura como um processo do comportamento humano, influenciado pela racionalidade, intuição e percepção, mostrando que a resolução de problemas não é feita apenas por meio de regras estabelecidas e nem sempre é possível ter acesso a todos os dados [Simon apud: Bertoncini et al. 2011]. Page (2002) trata da importância dos stakeholders no processo de tomada de decisão. Olender e Krenczyk (2017), pauta uma forma de encontrar o 
melhor custo-benefício na área de transpores através da teoria dos jogos. Pitt et al. (2005) sugere a tomada de decisão através da formalização de um protocolo de votação para organizações virtuais com vários agentes, governadas por normas. Manring (2007) apresenta um modelo que fornece base de conhecimento para auxiliar uma rede a compreender sua estrutura pelo diálogo, troca de informações/conhecimento e aprendizagem pelos stakeholders para, através do consenso, chegar a uma cultura de tomada de decisão colaborativa. Sarkis et al. (2007) indicam que um dos principais problemas de seleção de empresas parceiras depende muito das métricas de desempenho (tempo, custo, robustez e escopo) em agilidade que precisam ser atendidas pela outra empresa. Galeano et al. (2008) também utiliza o anteriormente citado ECOLEAD para auxiliar na tomada de decisão, com integração e monitoramento de indicadores de desempenho das partes envolvidas. De Lemos et al. (2015) descrevem a tomada de decisão no processo de seleção de parceiros para formação de organizações virtuais (VO). Após uma RSL, Lopez et al. (2019) sugerem o desenvolvimento de um framework cujo objetivo é apoiar empresas virtuais (VEs - Virtual Enterprise) em termos de Arquitetura Empresarial, melhorando a sinergia do grupo. Fang et al. (2008) apresentam um embasamento sobre empresas, tomada de decisão e confiança, e 9 hipóteses as quais são avaliadas com empresas chinesas, sendo possível observar que a confiança influencia a tomada de decisão. Zhang et al. (2010) propõem um algoritmo para verificar as possíveis junções de uma empresa em várias VOs, assim as organizações podem avaliar se devem ou não participar de uma VO. Retornando à ideia de aprendizagem trazida por Ganz (2018), pauta-se que as organizações tomam decisões em conflitos políticos, que levam à uma decisão inadequada. Calmet e Maret (2013) apresentam um framework que quantifica a confiança a partir de parâmetros matemáticos e auxilia então na tomada de decisão, demonstrando o quanto que a confiança é necessária para que ocorra uma decisão. Bhimani e Bromwich (2010) demonstram que pela economia globalizada, atual complexidade e rapidez do mercado é necessário envolver os atores da parte econômica (contadores) no processo de tomada de decisão e não somente pessoas envolvidas na linha gerencial.

Em termos de negociações, que envolve duas ou mais partes as quais buscam um objetivo através de uma decisão de consenso, o que leva à não aplicação de decisões unilaterais [Turel e Yuan 2010 apud: Oliveira e Camarinha-Matos 2010] e "benefícios para ambas as partes" [Simintiras e Thomas 1981 apud: Oliveira e Rodrigues 2005]. Hu et al. (2004) apresenta um framework com cooperação multiagente como foco. Haugland (2005) demonstram fatores que levam a uma negociação mais eficiente e que atende as partes envolvidas. Ryutov et al. (2017) apresentam um framework de suporte a VOs para criação de acordos através da captura do comportamento dos atores existentes. Zhao et al. (2006) estendem um workflow voltado para colaboração e contrato B2B (Business to Business) em VOs. Oliveira et al. (2008) e Oliveira e Camarinha-Matos (2008) detalham uma ferramenta, criada a partir do projeto ECOLEAD, de apoio à negociação através da formulação de contratos. Oliveira e Camarinha-Matos (2010) também pautam a importância do processo de negociação na formação de VOs, refletindo sobre modelos de acordo e propondo uma ferramenta de assistência à negociação para criação de VOs e estabelecimento de acordos. Obidallah et al. (2014) fornece a implementação da "estrutura de gerenciamento de mudança de procedimentos". Melin e Axelsson (2004) demonstram a simetria e assimetria existente numa "interação de negócios suportados por sistemas de informação interorganizacionais". Subramani e Venkatraman (2003) demonstram como 
fornecedores menores conseguem se resguardar com mecanismos de governança, integração e tomada de decisão perante empresas maiores. Rocha e Oliveira (2001) propõem um algoritmo de aprendizagem e demonstram um framework e um protocolo de negociação para auxiliar VOs. Bacarin et al. (2009) apresentam um framework para "uma estrutura de integração da cadeia produtiva agrícola". Torres et al. (2012) introduzem uma estratégia de negociação utilizada por agentes artificiais para medir a probabilidade de conflitos. Lazar (1997) traz um overview sobre parceria, benefícios e tipos de dificuldade encontradas (internas e externas) nessas parcerias.

No que tange as soluções/estratégias/ferramentas adotadas, o MSL indicou que não há uma ferramenta padrão, estratégia adotada comumente ou protocolo definido sobre qual ferramenta utilizar durante a gestão de conflitos, tomada de decisão e negociação. Além disso, 14 artigos fomentam uma solução baseada na literatura, em estratégia ou protocolo (classificados entre: proposta teórica, revisão sistemática, workflow, estratégia, overview e protocolo) e 19 artigos como proposta prática (classificados entre: algoritmo, modelo e framework). A necessidade da confiança em uma relação é um ponto estratégico, visto a quantidade de vezes e como aparece descrita na literatura. A cultura organizacional também foi levantada como uma forte vertente de diminuição de problemas, uma vez que existe um pensamento como um todo e tal parecer é voltado para o ganho da organização. Além disso, existem várias propostas as quais não foram passadas da fase de testes na realidade. Propostas para a tomada de decisão e negociação foram a maioria.

Por fim, sobre as limitações na tomada de decisão/gestão de conflitos/ negociação, a partir de uma análise global dos artigos, é possível inferir a falta de uma ferramenta padrão bem compreendida e formulada para apoiar o processo das empresas. Alguns autores levantaram a própria organização engajando seus funcionários, compreendendo sua cultura e compartilhando sua política internamente. Com essa perspectiva, é possível questionar até que ponto o apoio tecnológico é realmente a melhor solução para os problemas de conflito, decisão e negociação. Para uma ferramenta servir de apoio à operação, ela necessita ser bem compreendida e difundida pelos usuários, fácil de manipular e que atenda aos requisitos do negócio. Sem a sustentação dos utentes, uma solução tecnológica não cumpre o seu papel de facilitadora no processo, mas torna-se outro ponto burocrático no negócio. Para tal, o estudo de sistemas de informação, considerando suas 3 dimensões (organização, pessoas e tecnologia) [Laudon e Laudon 2016], torna-se essencial.

\section{Conclusão}

As organizações estão comumente relacionando-se entre si, buscando através dessa união seus interesses e mantendo-se dessa forma competitivas no mercado globalizado, dinâmico e abrupto. Nesse contexto, é frequente os conflitos, as negociações e consequentes tomadas de decisão.

O MSL demonstrou em que aspectos esses artigos compreendem em relação às questões de pesquisa definidas na Seção 2. Os resultados indicam uma falta de processo e de ferramenta voltada a gestão de conflitos, negociação e tomada de decisão (QP4 e QP5) que seja mainstream, ou seja, comum aos relacionamentos interorganizacionais e que auxilie as questões tratadas. Além disso, pode-se perceber as buscas para se encontrar um mecanismo para tal, como a quantidade de frameworks encontrados $(25 \%$ 
dos artigos). Acerca das questões QP1, QP2 e QP3, percebe-se uma maioria de artigos no tema (como conflitos relacionados a contratos), mas não uma resposta direta.

Em relação às ameaças à validade desta pesquisa, deve-se salientar que a string foi executada em bases que indexam literatura de Sistemas de Informação, e o uso de outras origens poderiam fornecer conhecimento e informação adicional aos resultados. Outro aspecto a ser considerado é o snowballing, o qual não retornou resultados consideráveis. Nesse contexto, pauta-se o quanto que a pesquisa perde em termos de amplitude de casos, sendo um desafio importante a extensão do tema.

Como trabalhos futuros, sugere-se a proposta de abordagens e ferramentas computacionais de apoio à gestão de conflitos, negociação e tomada de decisão em relacionamentos interorganizacionais, realizando experimentos em cenários reais para avaliar seu impacto e coletar dados que auxiliem em seu aprimoramento. Além disso, é possível refazer o MSL para atualizá-lo conforme a época.

\section{Agradecimentos}

Essa pesquisa foi apoiada pela UNIRIO [Edital PPInst-UNIRIO 2020].

\section{Referências}

Bacarin, E., Madeira, E.R.M., Medeiros, C. (2009) "Assembling and managing virtual organizations out of multi-party contracts", In: ICEIS Conference, pp.758-769.

Bertoncini C., Brito A., Leme E., Silva I. Silva T., Perri R. (2011) "Processo decisório: A tomada de decisão". Revista Científica Eletrônica de Agronomia, v.20, n.1.

Bhimani, A., Bromwich, M. (2010) "Management Accounting in a Digital and Global Economy: The Interface of Strategy, Technology, and Cost Information", In: Accounting, Organizations, and Institutions”, Oxford University Press, pp.85-111.

Biolchini, J., Mian, P.G., Natali, A.C. (2005) "Systematic Review in Software Engineering: Relevance and Utility”, Technical Report ES-679/05, PESC-UFRJ, RJ.

Bocanegra J., Pena J., Ruiz-Cortes A. (2011) "Interorganizational Business Modeling: An Approach for Traceability of Goals, Organizational Models and Business Processes". IEEE Latin America Transactions, v.9, n.1, pp.847-854.

Calmet, J., Maret, P. (2013) "Toward a Trust Model for Knowledge-Based Communities", In: WIMS Conference.

Carneiro, D., Novais, P., Lemos, F., Andrade, F., Neves, J. (2011) "Issues on conflict resolution in Collaborative Networks", In: IFIP AICT, pp.271-278.

de Lemos, F.S, Fiorese, A., Alves, O.C., Vieira, R.G. (2015) "Using data envelopment analysis and fuzzy logic as intelligent risk-based decision making support for virtual organizations”, In: Intelligent Systems Reference Library, pp.203-218.

Diirr B, Capelli C. (2018) "A systematic literature review to understand crossorganizational relationship management and collaboration”, In: HICSS Conference, pp.145-154.

Elliott, M.S., Scacchi, W. (2003) "Free Software Developers as an Occupational Community: Resolving Conflicts and Fostering Collaboration", ACM SIGGROUP Bulletin, pp.21-30. 
Fang, E.; Palmatier, R.W.; Scheer, L.K.; Li, N. (2008) “Trust at different organizational levels", In: ICEIS Conference, pp.80-98.

Galeano, N., Molina, A., Beeler, J., Monnier, F., Pouly, M., Aguilera, C., Olmo, A., Laessig, D., Tiefensee, B. (2008) "VBE pilot demonstrators", Methods and Tools for Collaborative Networked Organizations, pp.405-430.

Ganz, S.C. (2018) "Ignorant decision making and educated inertia: Some political pathologies of organizational learning”, Organization Science, pp.39-57.

Hu, J., Gao, J., Liao, B., Chen, J. (2004) “An agents based grid infrastructure of social intelligence”, In: AWCC Workshop, pp.33-38.

Kitchenham, B. (2004) "Procedures for Performing Systematic Reviews", Technical Report, Department of Computer Science, Keele University, Keele.

Lazar, F.D. (1997) "Partnering - New benefits from peering inside the black box", JMENEA Journal, pp.75-83.

Legner C., Wende K. (2007) "The Challenges of Interorganizational Business Process Design - a Research Agenda". In: ECIS Conference.

Lopez, C., Segura, M., Santórum, M. (2019) "Framework to Develop a Business Synergy through Enterprise Architecture”, In: ICISS Conference, pp.125-129.

Malakowsky, H., Kassick C. (2014) "O conflito no ambiente de trabalho: Um estudo sobre causas e consequências nas relações interpessoais", Revista Gestão e Desenvolvimento, v.11, n.1.

Manring, S.L. (2007) "Creating and managing interorganizational learning networks to achieve sustainable ecosystem management", Organization and Environment, pp.325-346.

Melin, U., Axelsson, K. (2004) "Emphasizing Symmetry Issues in Business Interaction Analysis and IOS”, In: ICEC Conference, pp.312-318.

Ness, H., Haugland, S.A. (2005) "The evolution of governance mechanisms and negotiation strategies in fixed-duration interfirm relationships", JBR Journal, pp.1226-1239.

Obidallah, W.J., Raahemi, B., Alaieri, Fahad S. (2014) "Change processes and Procedures in Service Oriented Virtual Organizations and Collaborative Network", In: MEDES Conference, pp.50-55.

Olender, M., Krenczyk, D. (2017) "Practical example of game theory application for production route selection", In: IOP Conference Series.

Oliveira, A.I., Camarinha-Matos, L.M. (2010) "Negotiation and Contracting in Collaborative Networks", In: Emerging Trends in Technological Innovation, pp.8392.

Oliveira, A.I., Camarinha-Matos, L.M., Pouly, M. (2008) "Agreement negotiation support in VO creation", In: PRO-VE Conference, pp. 107-118.

Oliveira, A.I., Camarinha-Matos, L.M. (2008) "Agreement negotiation wizard", Methods and Tools for Collaborative Networked Organizations, pp.191-218. 
Oliveira, J.V., Rodrigues, L.C. (2005) "O Processo de Negociação, sua Estrutura e Importância no contexto Atual”.

Ollus, M., Jansson, K., Karvonen, I. (2007) "On the management of collaborative SME networks and Contracting in Collaborative Networks", In: IFAC-PapersOnline, pp.49-54.

Page, C.G. (2002) "The determination of organization stakeholder salience in public health", JPHMP Journal, pp.76-84.

Pitt, J., Kamara, L., Sergot, M., Artikis, A. (2005) "Formalization of a Voting Protocol for Virtual Organizations”, In: AAMAS Conference, pp.373-380.

Rocha, A.P., Oliveira, E. (2001) "Electronic institutions as a framework for agents' negotiation and mutual commitment”, In: EPIA Conference, pp.232-245.

Ryutov, T., Neuman, C., Zhou, L., Foukia, N. (2005) "Establishing agreements in dynamic virtual organizations", In: SecureComm Conference, pp.92-101.

Samdantsoodol, A., Cang, S., Yu, H. (2012) "Overview of virtual enterprises in supply chain management", In: ICAC Conference, pp.193-201.

Sarkis, J., Talluri, S., Gunasekaran, A. (2007) "A strategic model for agile virtual enterprise partner selection”, IJOPM Journal, pp.1213-1234.

Subramani, M.R., Venkatraman, N. (2003) "Safeguarding investments in asymmetric interorganizational relationships: Theory and evidence", AMJ Journal, pp.46-62.

Tayles, M., Webster, M., Sugden, D., Bramley, A. (2005) "Accounting "gets real" in dealing with virtual manufacturing”, JIC Journal, pp.322-338.

Torres, R., Rivera, D., Astudillo, H. (2012) "Web service compositions which emerge from virtual organizations with fair agreements", In: AMSTA Conference, pp.34-43.

Troussier, N., Belkadi, F., Eynard, B., Messaadia, M., Gidel, T. (2010) "Supplieroriented and product life cycle management framework to support virtual organisations", IJPD Journal, pp.49-66.

Van Fenema, P., Keers, B., Zijm H. (2014) "Interorganizational Shared Services: Creating Value across Organizational Boundaries". Shared Services as a New Organizational Form, v.13, pp.175-217.

Wholin, C. (2014) "Guidelines for Snowballing in Systematic Literature Studies and a Replication in Software Engineering”, In: EASE Conference.

Zhang, G., Jiang, J., Su, Z., Qi, M., Fang, H. (2010) "Searching for overlapping coalitions in multiple virtual organizations", Information Sciences, pp.3140-3156.

Zhao, F., Zhang, Q., Yu, D., Chen, X., Yang, Y. (2005) “A hybrid algorithm based on PSO and simulated annealing and its applications for partner selection in virtual enterprise", In: ICO Conference, pp.380-389.

Zhao, X., Liu, C., Yang, Y. (2006) "Supporting Virtual Organisation Alliances with Relative Workflows", In: APCCM Conference, pp.115-124.

Zhu Z., Huang H. (2007) "The Cultural Integration in the Process of Cross-border Mergers and Acquisitions". IMR Journal, v.3, n.2, pp.40-44. 